\title{
Configuration Property of the Chinese First Quasi-Axisymmetric Stellarator ${ }^{*}$
}

\author{
Akihiro SHIMIZU ${ }^{1)}$, Haifeng LIU ${ }^{2)}$, Mitsutaka ISOBE ${ }^{1,3)}$, Shoichi OKAMURA ${ }^{1)}$, \\ Shin NISHIMURA ${ }^{1)}$, Chihiro SUZUKI ${ }^{1)}$, Yuhong $\mathrm{XU}^{2)}$, Xin $\mathrm{ZHANG}^{2)}$, Bing $\mathrm{LIU}^{2)}$, \\ Jie $\mathrm{HUANG}^{2)}$, Xiangqu WANG ${ }^{2)}$, Hai LIU ${ }^{2)}$, Changjian TANG ${ }^{2,4)}$ and CFQS team ${ }^{1,2)}$ \\ ${ }^{1)}$ National Institute for Fusion Science, National Institutes of Natural Sciences, Toki 509-5292, Japan \\ ${ }^{2)}$ Institute of Fusion Science, School of Physical Science and Technology, Southwest Jiaotong University, \\ Chengdu 610031, China \\ ${ }^{3)}$ SOKENDAI (The Graduate University for Advanced Studies), Toki 509-5292, Japan \\ ${ }^{4)}$ School of Physical Science and Technology, Sichuan University, Chengdu 610041, China
}

(Received 11 January 2018 / Accepted 7 October 2018)

The Chinese First Quasi-axisymmetric Stellarator (CFQS) is a new quasi-axisymmetric experimental device planned for construction at South West Jiaotong University (SWJTU), China. This is a joint project of the National Institute for Fusion Science (NIFS) and the SWJTU. The present paper discusses the equilibrium configuration of the CFQS with a major radius of $1.0 \mathrm{~m}$, a toroidal magnetic field strength of $1.0 \mathrm{~T}$, and an aspect ratio of 4.0. As the CFQS is a quasi-axisymmetric stellarator, a tokamak-like bootstrap current is expected. The magnitude of the bootstrap current was estimated by BOOTSJ code. Next, the effects of the bootstrap current on the quasi-axisymmetric property and the neoclassical diffusion coefficient were estimated. The bootstrap current little affected the quasi-axisymmetric property, and a good neoclassical transport property was maintained.

(C) 2018 The Japan Society of Plasma Science and Nuclear Fusion Research

Keywords: quasi-axisymmetric stellarator, modular coils, low aspect ratio, optimized, CFQS, CHS-qa

DOI: $10.1585 /$ pfr. 13.3403123

\section{Introduction}

The magnetic field configuration of a helical device is produced by an external magnetic field coil system. The steady-state operation of such devices is considered to be advantageous for future nuclear fusion reactors. However, the neoclassical transport in conventional stellarators is not good in the collision-less regime (the so-called $1 / v$ regime, where $v$ is the collision frequency), because the diffusion coefficient is proportional to $1 / v$ and the neoclassical transport degrades in reactors with relevant plasma parameters.

Various optimized stellarator configurations have been proposed and constructed as real experimental devices during the last several decades. Examples are the Helically Symmetric Experiment (HSX) [1,2] and Wendelstein 7-X (W7X) [3], which have optimal magnetic field configurations, improved neoclassical transport in the $1 / v$ regime, and stable magnetohydrodynamics (MHD) properties. A quasi-axisymmetric stellarator (QAS) is an optimized stellarator with an axisymmetric magnetic configuration in magnetic (i.e., Boozer) coordinates $[4,5]$. Its neoclassical transport properties are similar to those of tokamaks, but without requiring an inductively driven current. Therefore, the QAS is a tokamak-like stellarator that combines the advantages of both stellarators and tokamaks.

author'se-mail: akihiro@nifs.ac.jp

*) This article is based on the presentation at the 26th International Toki Conference (ITC26).
One future QAS is the Chinese First Quasiaxisymmetric Stellarator (CFQS), which will be constructed at the South West Jiaotong University (SWJTU) in China. This project (called NSJP) is a conjoint effort between the National Institute for Fusion Science (NIFS) and the SWJTU. Cooperative experiments by both organizations will be conducted on the CFQS.

The physics and engineering aspects of this device are currently being designed. The proposed parameters of the device are as follows: toroidal magnetic field $B_{\mathrm{t}}=1.0 \mathrm{~T}$, major radius $R=1.0 \mathrm{~m}$, and aspect ratio $A_{\mathrm{p}}=4.0$. This paper presents the equilibrium configuration of the CFQS, then examines the bootstrap current and its effect on the quasi-axisymmetric configuration.

\section{Equilibrium and Modular Coil System of the CFQS}

The present equilibrium configuration of CFQS is based on the CHS-qa, 2 b32 configuration [6] (aspect ratio $=3.2$ ), which was previously considered for a postCompact Helical System (CHS) project. Based on this configuration, the geometry of the outermost magnetic surface of the CFQS was determined, therefore, the toroidal periodic number of the CFQS was chosen as 2. Difficulties are expected in fabricating the modular coil system of a compact machine with a low aspect ratio (e.g., 


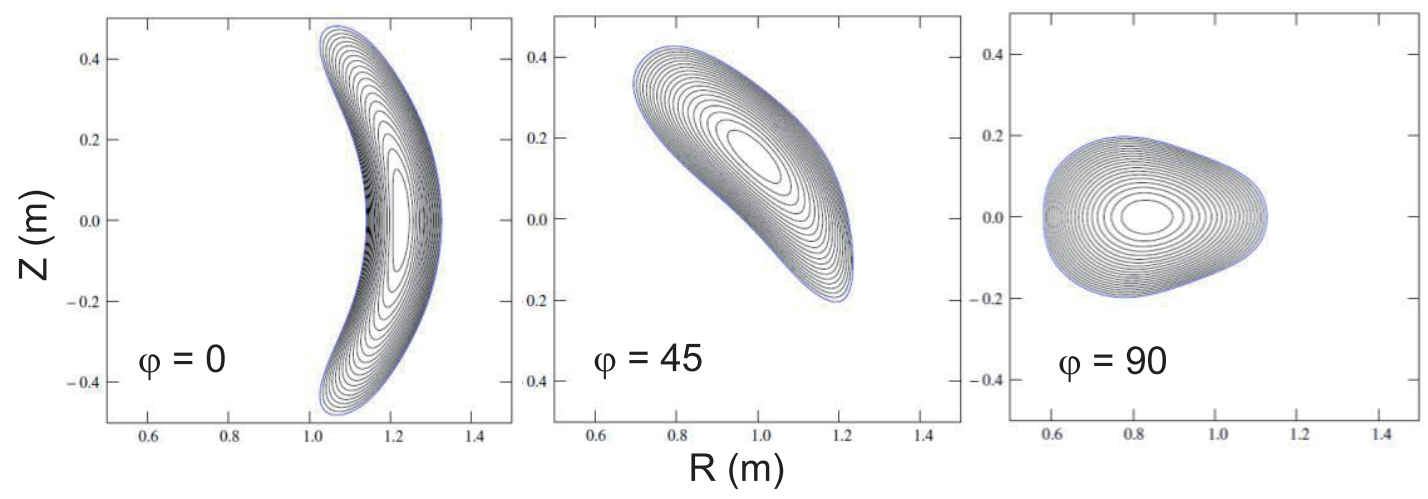

Fig. 1 Poloidal cross sections of the CFQS equilibrium calculated by VMEC free-boundary at three toroidal angles.

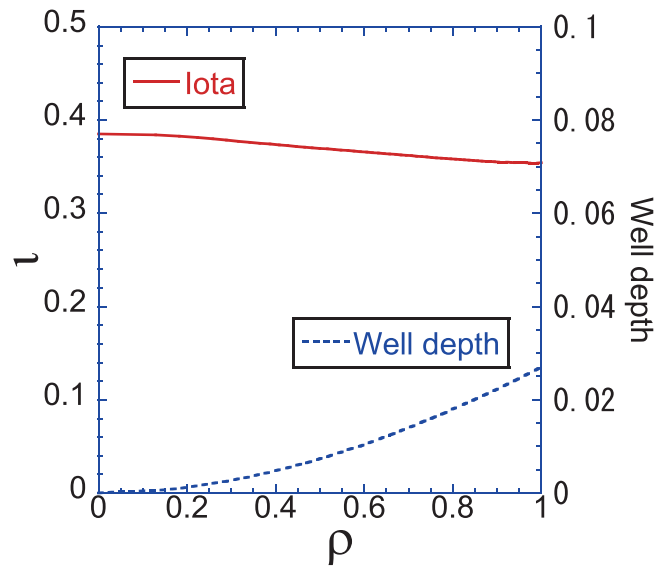

Fig. 2 Radial profiles of the rotational transform and well depth of the CFQS at $\beta=0.0 \%$.

$R=1.0 \mathrm{~m}$ ). Therefore, the aspect ratio of the CFQS was enlarged to 4.0. As in other optimized stellarators [7, 8], the modular coil system was optimized to realize the target geometry of the outermost magnetic surface, which was determined before designing the magnetic coil system. The modular coil system produces an average normalized normal component of the magnetic field, $\mathbf{B} \cdot \mathbf{n} /|\mathrm{B}|$, on the target outermost magnetic surface. The coil optimization process reduces this component to zero, while simultaneously engineering the properties of the filament coils, such as the minimum distance between the coils and the minimum curvature radius of the coils are also evaluated and optimized. The modular coil system was optimized by the NESCOIL code $[9,10]$. In total, the CFQS contains 16 modular coils. The minimum coil-to-coil distance and radius of curvature of the coils are $18.5 \mathrm{~cm}$ and $21.5 \mathrm{~cm}$, respectively (estimated for filament structure coils [11]). The free-boundary VMEC [12] equilibrium results for the $0 \%$ volume-averaged beta, $\beta$, at different toroidal angles $\left(0^{\circ}, 45^{\circ}\right.$ and $\left.90^{\circ}\right)$ are shown in Fig. 1 . Figure 2 shows the radial profiles of the rotational transform and magnetic well depth. The typical rotational transform was 0.4 , and the profile is a weak shear profile as obtained in other optimized stellarators. The magnetic well property was achieved throughout the region.

\section{Operation Parameter Regime Estimated from the ISS95 Scaling Law}

The operation parameters of CFQS were estimated using the ISS95 scaling law [13]. The scaling law is written as follows:

$$
\tau_{\text {ISS95 }}=0.079 a^{2.21} R^{0.65} P^{-0.59} \bar{n}_{\mathrm{e}}^{0.51} B^{0.83} t_{2 / 3}^{0.4}
$$

The parameters (units) in Eq. (1) are the energy confinement time $\tau(\mathrm{s})$, the minor radius $a(\mathrm{~m})$, the major radius $R$ (m), the heating power $P$ (MW), the line-averaged density $\bar{n}_{\mathrm{e}}\left(10^{19} \mathrm{~m}^{-3}\right)$, the toroidal magnetic field $B(\mathrm{~T})$, and the rotational transform $t$ at $\rho=2 / 3$ (dimensionless). The radial density and temperature profiles are assumed as $T=T_{0}$ $\left(1-\rho^{2}\right)$ and $n_{\mathrm{e}}=n_{\mathrm{e} 0}\left(1-0.8 \rho^{2}+1.3 \rho^{2}-1.5 \rho^{3}\right)$, respectively. The values of $a, R, B$, and $t_{2 / 3}$ were fixed at $0.25 \mathrm{~m}, 1.0 \mathrm{~m}$, $1.0 \mathrm{~T}$, and 0.35 respectively. In the first case, $T_{\mathrm{i} 0}=0.75$ $T_{\mathrm{e} 0}$ and $\bar{n}_{\mathrm{e}}=0.78 \times 10^{19} \mathrm{~m}^{-3}\left(n_{\mathrm{e} 0}=1.0 \times 10^{19} \mathrm{~m}^{-3}\right)$ were assumed. Figure 3 plots the estimated electron temperature as a function of heating power for three $\mathrm{H}$ factors $\left(H_{\mathrm{f}}\right)$. The $H_{\mathrm{f}}$ defines the improved factor of the confinement time. The expected $T_{\mathrm{e} 0}$ ranged from 3.0 to $4.6 \mathrm{keV}$ at a heating power of $1 \mathrm{MW}$.

\section{Bootstrap Current and Its Effect on the Configuration}

The neoclassical properties of the QAS resemble those of tokamaks. Therefore similarly to the CHS-qa [14], a large bootstrap current is expected in the QAS. The magnitude of the bootstrap current was estimated by BOOTSJ code, which uses a semi-analytic formula in the collision-less limit [15]. The temperature and density profiles were assumed as $T=T_{0}\left(1-\rho^{2}\right)$ and $n_{\mathrm{e}}=n_{\mathrm{e} 0}$ $\left(1-0.8 \rho^{2}+1.3 \rho^{4}-1.5 \rho^{6}\right)$, respectively, and $T_{\mathrm{i} 0}$ was set 


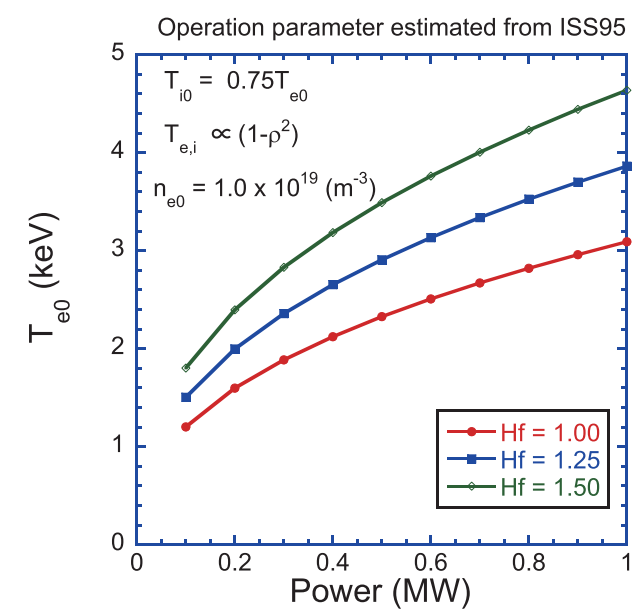

Fig. 3 Plasma parameters estimated by the ISS95 scaling law with $T_{\mathrm{i} 0}=0.75 T_{\mathrm{e} 0}$, and $n_{\mathrm{e} 0}=1.0 \times 10^{19} \mathrm{~m}^{-3}$.

to $0.75 T_{\mathrm{e} 0}$. Figure 4 (a) shows the radial profiles of $n_{\mathrm{e}}, T_{\mathrm{e}}$, and $T_{\mathrm{i}}$, and Fig. 4 (b) shows the normalized collisionality values $v^{*}$ and $v^{* *}$ with $n_{\mathrm{e} 0}$ and $T_{\mathrm{e} 0}$ fixed at $1 \times 10^{19} \mathrm{~m}^{-3}$ and $3.46 \mathrm{keV}$, respectively. Here $v^{*}$ and $v^{* *}$ are the collisionality $v_{\mathrm{ei}}$ normalized by the toroidal banana orbit frequency $\left(v_{\mathrm{b}}=\varepsilon_{\mathrm{t}}^{3 / 2} t / R \cdot\left(T_{\mathrm{e}} / m_{\mathrm{e}}\right)^{1 / 2}\right)$ and the helical banana orbit frequency $\left(v_{\mathrm{hb}}=\left(\varepsilon_{\mathrm{h}} / \varepsilon_{\mathrm{t}}\right)^{3 / 4} v_{\mathrm{b}}\right)$, respectively. The parameters $\varepsilon_{\mathrm{t}}, \varepsilon_{\mathrm{h}}$, and $t$ denote the toroidal ripple, helical ripple, and rotational transform, respectively. Here, for $\varepsilon_{\mathrm{h}}$, effective herical ripple is used.

The radial profile of the bootstrap current at $\beta=$ $1.19 \%$ is shown in Fig. 5. The equilibrium calculation was obtained by VMEC free-boundary calculation, assuming the modular coil system described in Section 2. The total bootstrap current in this case (with $\beta=1.19 \%$ ) was evaluated as $26.1 \mathrm{kA}$. Figure 6 shows the radial profile of the Fourier components of the magnetic field strength $B$ (expressed as $B=\Sigma B_{\mathrm{mn}} \cos (m \theta-n N \varphi)$ in Boozer coordinates) for $\beta=0.0 \%$ and $\beta=1.19 \%$. The dominant $B_{10}$ can be explained by the axisymmetric configuration. The absolute values of the non-axisymmetric components were below $0.02 \mathrm{~T}$, and the Shafranov shift made no significant effect on the quasi-axisymmetric property. The bootstrap current is plotted as a function of $\beta$ in Fig. 7. The bootstrap current was proportional to $\beta$, and reached approximately $35 \mathrm{kA}$ at $\beta=1.5 \%$.

The effect of bootstrap current on the neoclassical transport was investigated by NEO code [16], which estimates the radial profile of the effective helical ripple, $\varepsilon_{\mathrm{eff}}$. The neoclassical diffusion coefficient $D_{\text {neo }}$ of the stellarators in the $1 / v$ regime is related to $\varepsilon_{\mathrm{eff}}$ as follows:

$$
D_{\text {neo }} \propto \frac{v_{\mathrm{d}}^{2} \varepsilon_{\mathrm{eff}}^{3 / 2}}{v} .
$$

Here, $v$ and $v_{\mathrm{d}}$ are the collision frequency and the drift velocity, respectively. As $D_{\text {neo }}$ is proportional to $\varepsilon_{\text {eff }}^{3 / 2}$, it is an effective comparative indicator for various configurations.

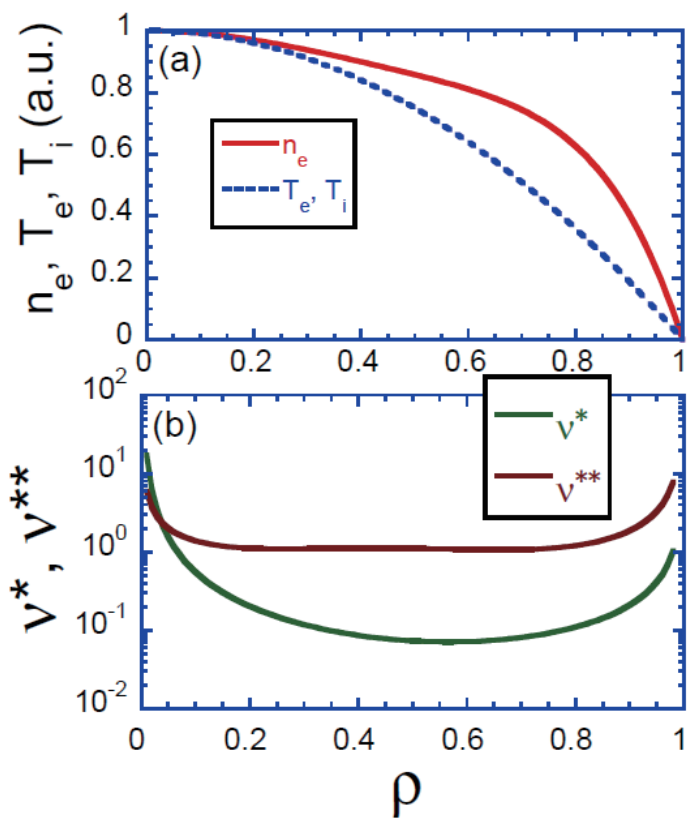

Fig. 4 (a) Assumed radial profiles of $n_{\mathrm{e}}, T_{\mathrm{e}}$, and $T_{\mathrm{i}}$. (b) Electronion collisionality normalized by the toroidal banana frequency $\left(v^{*}\right)$ and the helical banana frequency $\left(v^{* *}\right)$, with $n_{\mathrm{e} 0}=1 \times 10^{19} \mathrm{~m}^{-3}$ and $T_{\mathrm{e} 0}=3.46 \mathrm{keV}$.

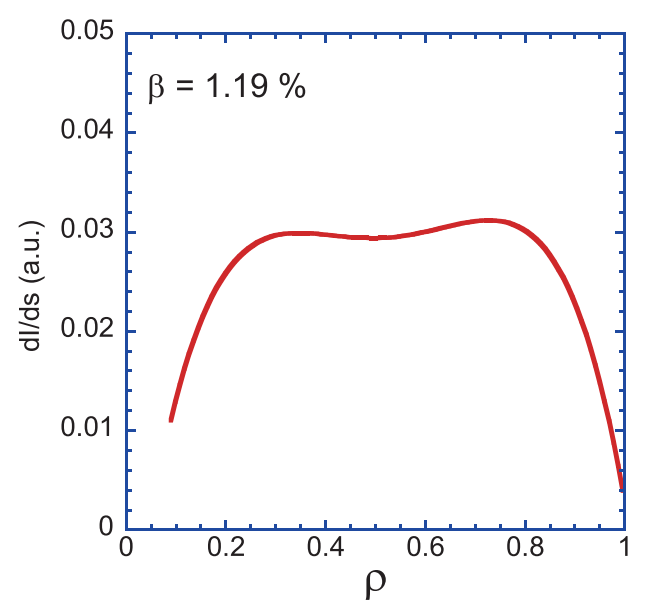

Fig. 5 Radial bootstrap-current profile in the CFQS at volume averaged $\beta=1.19 \%$, estimated by BOOTSJ code. The temperature and density profiles are assumed as $T=T_{0}$ $\left(1-\rho^{2}\right)$ and $n_{\mathrm{e}}=n_{\mathrm{e} 0}\left(1-0.8 \rho^{2}+1.3 \rho^{4}-1.5 \rho^{6}\right)$, respectively, and $S=\rho^{2}$.

The radial profiles of $\varepsilon_{\mathrm{eff}}^{3 / 2}$ estimated by the NEO code are shown in Fig. 8. This figure shows the radial profiles of $\varepsilon_{\text {eff }}$ for the free-boundary VMEC equilibrium in the CFQS for various beta (accounting for the bootstrap current). The $\varepsilon_{\mathrm{eff}}^{3 / 2}$ profile of the fixed boundary VMEC equilibrium in CHS $\left(R_{\mathrm{ax}}=92.1 \mathrm{~cm}, \beta=0.0 \%\right)$ [17] is also shown as a reference. The $\varepsilon_{\text {eff }}^{3 / 2}$ was approximately three orders of magnitude lower in CFQS than in CHS. The neoclassical transport maintained a good confinement property as $\beta$ increased. 
(a)

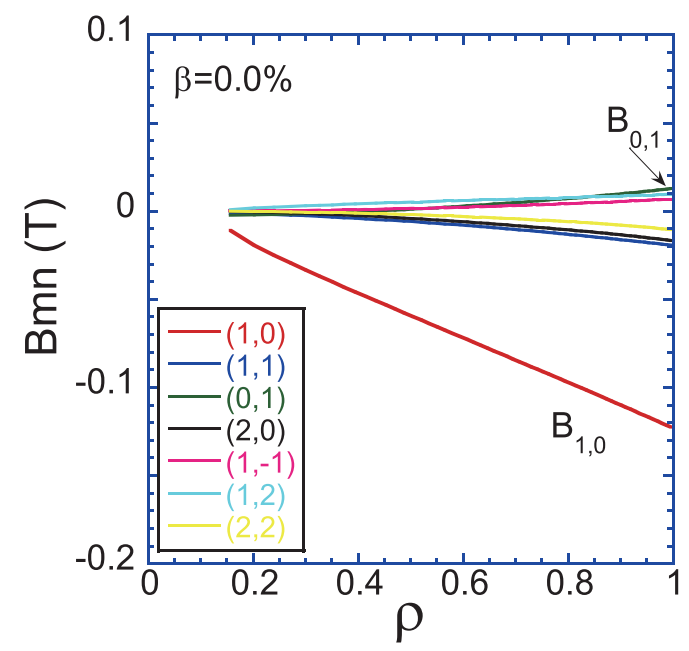

(b)

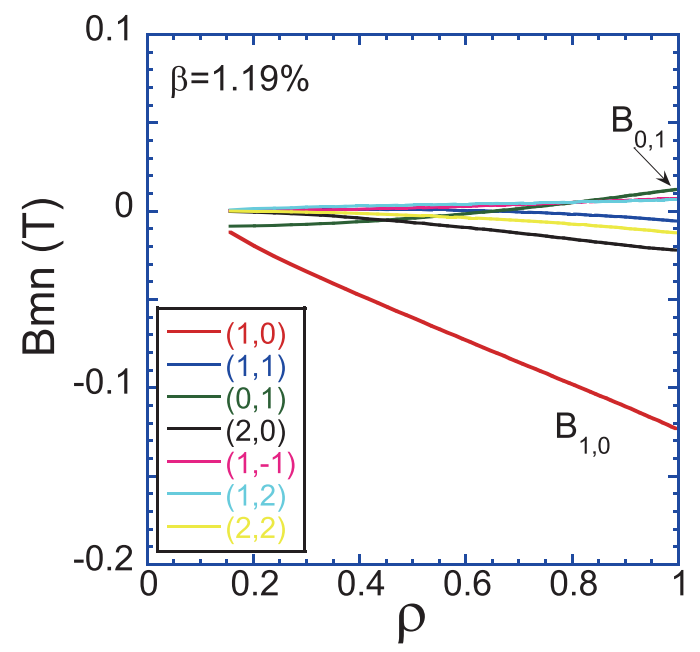

Fig. 6 Fourier spectra of the CFQS magnetic-field configuration, obtained by VMEC free-boundary calculation in Boozer coordinates: (a) $\beta=0.0 \%$ and (b) $\beta=1.19 \%$.

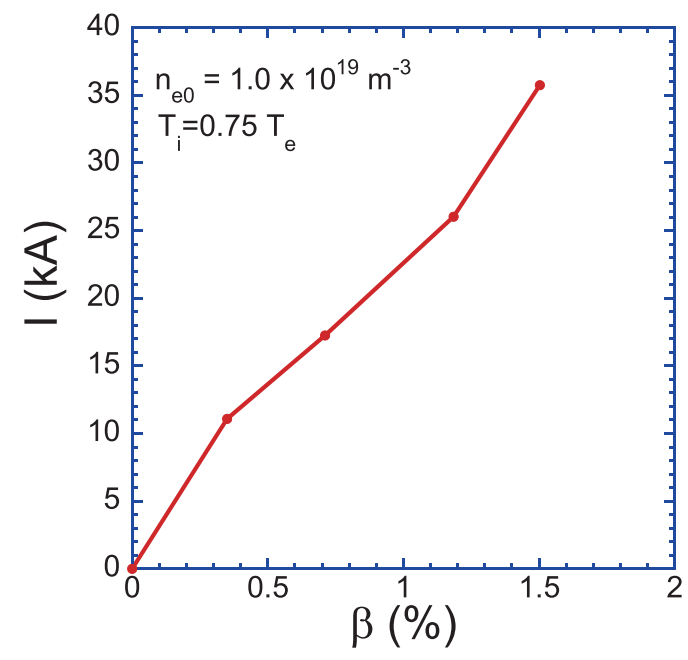

Fig. 7 Bootstrap current versus volume-averaged $\beta$ in the CFQS.

In the quasi-axisymmetric CFQS configuration, increasing the bootstrap current increased the rotational transform, as observed in tokamaks. The radial profiles of the rotational transform for various beta are shown in Fig. 9. As beta increased, the rotational transform crossed rational values, e.g., $0.4,0.5$. The effect of changing the rotational transform must be checked in a stability analysis. This analysis will be implemented by ideal MHD calculation code, such as TERPSICHORE [18].

\section{Summary}

This paper presented the current equilibrium configuration $(R=1.0 \mathrm{~m}, B=1.0 \mathrm{~T})$ of the CFQS. The operation regime was estimated from the ISS95 scaling law, and the bootstrap current (reaching $35 \mathrm{kA}$ at $\beta=1.5 \%$ ) was estimated by BOOTJ code on the VMEC free-boundary equi-

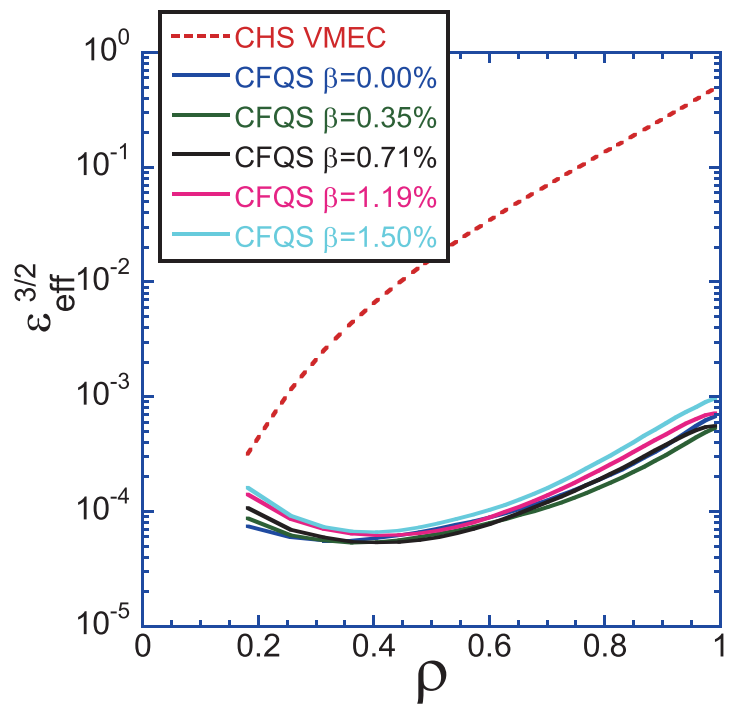

Fig. 8 Radial profiles of effective helical ripple in the CFQS for various $\beta$ with bootstrap current. Results were obtained from the VMEC free-boundary calculation. The CHS result ( $R_{\mathrm{ax}}=92.1 \mathrm{~cm}, \beta=0.0 \%$; red dashed curve) is shown as a reference.

librium. The effect of the bootstrap current on the quaxiaxisymmetry and neoclassical transport was then investigated. The bootstrap current effect is not large and good neoclassical transport properties are maintained up to an average $\beta$ of $1.5 \%$, as confirmed by NEO code. A detailed stability analysis using MHD code will be undertaken in future work.

\section{Acknowledgement}

The authors are thankful for strong support and encouragement to this project (NSJP) from Director Gen- 


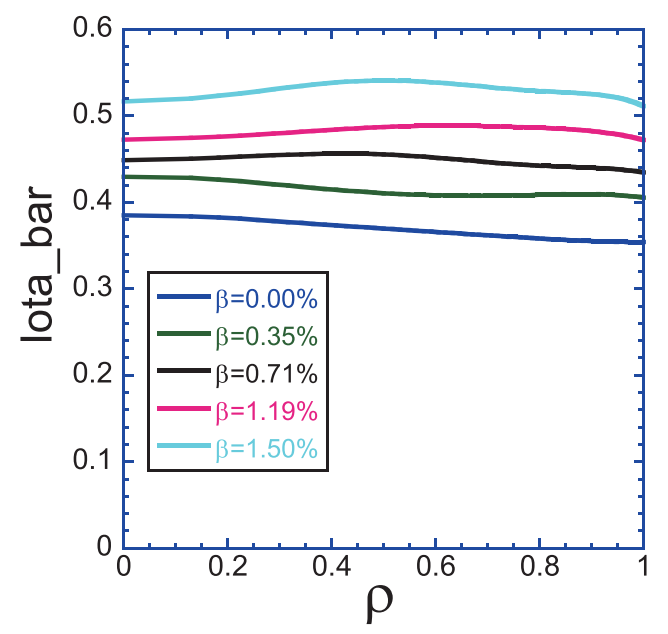

Fig. 9 Radial profiles of rotational transforms of the CFQS for various $\beta$, accounting for the bootstrap-current effect.

eral Prof. Y. Takeiri of NIFS and Vice President Prof. W.G. Zhang of SWJTU. The authors are also grateful for the support to the NSJP from other related staff of NIFS and SWJTU. This research is supported by NIFS general collaboration project, budget number NIFS17KBAP034, NIFS17KLPP048, NIFS budget URSX401, and also supported by JSPS KAKENHI Grant No. 16K06946.
[1] F.S.B. Anderson et al., Trans. Fusion Tech. 27, 273 (1995).

[2] D.T. Anderson et al., J. Plasma Fusion Res. SERIES 1, 49 (1998).

[3] T. Klinger, A. Alonso, S. Bozhenkov et al., Plasma Phys. Control. Fusion 59, 014018 (2017).

[4] A.H. Boozer, Phys. Fluids 24, 1999 (1981).

[5] A.H. Boozer, Phys. Fluids 23, 904 (1980).

[6] S. Okamura, K. Matsuoka, S. Nishimura, M. Isobe et al., Nucl. Fusion 41, 1865 (2001).

[7] J. Nuhrenberg and R. Zille, Phys. Lett. A 129, 113 (1988).

[8] P. Garabedian, Phys. Plasmas 3, 2483 (1996).

[9] M. Dravlak, Fusion Technol. 33, 106 (1998).

[10] P. Merkel, Nucl. Fusion 27, 867 (1987).

[11] H. Liu et al., Plasma Fusion Res. 13, 3405067 (2018).

[12] S.P. Hirshman and J.C. Whitson, Phys. Fluids 26, 3553 (1983).

[13] U. Stroth, M. Murakami, R.A. Dory et al., Nucl. Fusion 36, 1063 (1996).

[14] M. Isobe, S. Okamura, N. Nakajima et al., J. Plasma Fusion Res. SERIES 5, 360 (2002).

[15] K.C. Shaing et al., Phys. Fluids B1, 148 (1989).

[16] V.V. Nemov, S.V. Kasilov et al., Phys. Plasmas 6, 4622 (1999).

[17] K. Matsuoka et al., 12th Int. Conf. Plasma Phys. and Control. Nucl. Fusion Research, Nice, 1988 (IAEA, Vienna) Vol.2, p.411 (1988).

[18] G.Y. Fu, W.A. Cooper, R. Gruber et al., Phys. Fluids B4, 1401 (1992). 